\title{
UNDERSTANDING HOW TESLA LEVERAGES TWITTER TO COMMUNICATE ENVIRONMENTAL CORPORATE SOCIAL RESPONSIBILITY
}

\author{
by \\ Kirstin Corbett \\ BA Communications and Anthropology, \\ Wilfrid Laurier University 2019
}

\author{
A MRP \\ presented to Ryerson University
}

In partial fulfillment of the requirements for the degree of

Master of Professional Communication

Toronto, Ontario, Canada, 2020

(C) Kirstin Corbett, 2020 


\section{Table of Contents}

Abstract $\quad$ iii

Introduction 1

$\begin{array}{ll}\text { Literature Review } & 2\end{array}$

Environmental Corporate Social Responsibility (ECSR) 2

Car Culture and the Use of Nature in Green Marketing 4

$\begin{array}{ll}\text { ECSR and Tesla's Twitter } & 7\end{array}$

$\begin{array}{lr}\text { Research Questions } & 8\end{array}$

$\begin{array}{lr}\text { Methodology } & 9\end{array}$

$\begin{array}{ll}\text { Data Collection Steps } & 10\end{array}$

$\begin{array}{ll}\text { Drawbacks } & 12\end{array}$

Rhetorical analysis $\quad 13$

$\begin{array}{ll}\text { Relation to Stock Price } & 15\end{array}$

$\begin{array}{ll}\text { Content Analysis } & 17\end{array}$

$\begin{array}{ll}\text { Conclusion } & 22\end{array}$

References $\quad 25$

$\begin{array}{lr}\text { Appendix } & 29\end{array}$ 


\begin{abstract}
As the media coverage of the global climate crisis grows, so does the act of climate conscious consumer activism. As a result, companies have turned to communicating their corporate social responsibility (CSR) on social media in order to leverage their sustainable activities and improve their corporate image. Twitter, specifically, is a popular social media platform for marketing and cultivating business to consumer (B2C) relationships. One company that has become a model of success for CSR on Twitter is Tesla. The findings of this study discover higher interaction around ECSR tagged tweets in combination with a high culture of sharing amongst Tesla's followers. This study aims to discover how Tesla, an electric car and sustainable energy company, has made their Twitter page both informative and interactive when communicating their environmental corporate social responsibility (ECSR) agenda online.
\end{abstract}

Keywords: Environmental Corporate Social Responsibility, Tesla, Twitter, Online Community 


\section{Understanding how Tesla Leverages Twitter for ECSR}

Introduction

Given the increasing concerns regarding climate change, consumer activism has forced large industries to double down on their efforts related to corporate social responsibility (CSR) communication (Bubna-Litic, K, 2007). Corporate social responsibility is a type of business selfregulation used by large companies to follow philanthropic initiatives that are socially and environmentally responsible. These days, social media serves as a CSR communication tool that companies can use to strengthen the overall organization-public relationship (OPR) which is often formed through brand loyalty over time.

One company has arguably found the magic key for unlocking successful CSR initiatives online -- Tesla, Inc. -- currently the world's most valuable automotive company. Tesla goes to enormous efforts on Twitter to communicate environmental CSR (ECSR) to their 6.1 million followers and is a great example of how a car company can justify its existence using social media in the era of climate change. The symbolic construction of Tesla online is, of course, reinforced and affirmed by the responses from their audience on Twitter.

This study will examine Tesla's use of ECSR on Twitter (excluding Elon Musk's personal Twitter feed as a super influencer) in order to understand the ways corporations can successfully market ECSR online. By providing a space where people can share photos and information about the products, Tesla has been successful communicating ECSR online. This study will begin by conducting a rhetorical analysis of Tesla's use of Twitter, then delve into a content analysis and a social network analysis of Tesla's ECSR tweets to understand how Tesla leverages Twitter for their ECSR communication. 
Literature Review

Environmental Corporate Social Responsibility (ECSR)

Corporate social responsibility (CSR) communication is a key tool for supporting environmental change at an organizational level. In 2009, the World Bank defined the environmental expectations of CSR as: 1) eliminating waste, 2) maximizing productivity, 3) protecting the environment, and 4) minimizing the effects of production for future generations (World Bank International Finance Committee, 2009, p. 2). CSR can be broken down into both internal CSR and external CSR. Internal CSR is focused on the well-being of employees and their emotional connection to the organization, while external CSR is focused on the environmental and social practices of an organization and the ways these efforts can strengthen its reputation.

Some organizations refer to sustainability in their main mission statement, but it is often difficult to assess a firm's total sustainability impacts since there are often no standardized CSR or sustainability metrics with which to analyze the external and internal information communicated to stakeholders (Vigneau, Humphreys, and Moon, 2015, p.469). Having said that, there is a non-profit Global Reporting Initiative (GRI) founded by the United Nations Environment Program (UNEP), Ceres and the Tellus Institute that encourages companies to disclose their sustainability performance using financial metrics. Regardless of how firms use external CSR communication, it is important to recognize that "external sustainability reporting offers a marketing opportunity that can build a strong corporate image and reputation" (Reilly and Larya, 2018, p. 622).

When assessing the sustainability performance of corporations, a balanced scorecard approach to their CSR is necessary. The most popular approach is the triple bottom line approach 
which examines: 1) the environmental impact or effect on the physical environment, 2) economic sustainability or viability to the economic system, and 3) social sustainability or the impact of the corporation's philanthropy on the consumer or community (Sheth, Sethia, and Srinivas, 2011, p.20). CSR corporate communication can be communicated formally, through CSR reports, or informally, through, for example, social media. One study in 2014 took a sample of 16 global companies from four different industries of both their formal communications and their social media platforms (Facebook and Twitter) and discovered that so-called "green" companies (such as Tesla) discussed sustainability more within their corporate communications and were more active on social media to build brand awareness and share eco-friendly accomplishments (Reilly and Hynan, 2014, p. 748). This study confirms that corporate communication in terms of sustainability or environmental corporate social responsibility (ECSR) uses social media as a key outlet for CSR communication in order to share content and connect with their consumers. Not only is CSR a means of communicating responsible and ethical business practices to targeted audiences, it is also a powerful tool when used in combination with social media (Gharios, Yahchouchi, and Chaker, 2020, p.18).

With the increase of environmental crises ostensibly influenced by human consumption, there is a growing need for companies, especially green companies, to account for their environmental impacts and actions. In order to build long term relationships with both external and internal stakeholders of a company, communicating environmental initiatives are essential to economic growth (Rashid, 2014, p.502). To build a green organization, a company must be aware of the potential harm that their products may have on future generations, and how to minimize their impacts and emissions. This is where environmental corporate social responsibility (ECSR) comes in to maintain a company's overall image so they can be held 
accountable for their actions and strengthen the relationship with their consumers. Environmental customer welfare, environmental community involvement, and environmental philanthropy are the key dimensions of ECSR that companies follow to successfully convey their environmental intentions. With the rise of social media, companies' ability to communicate about their ECSR efforts is no longer reserved for impact reports and commercials, but can also be communicated through online platforms like Twitter.

\section{Car Culture and the Use of Nature in Green Marketing}

In American culture, the car affords mobility and is considered a necessity due to a lack of public transportation infrastructure and because it promises the freedom of the open road. The naturalization of private vehicle ownership serves as a symbol of modern life and represents privacy, mobility, power, and status (Chen, 2016, p. 12). Within this cultural context, there has been an increase in automobile companies promoting energy efficiency in order to promote green consumerism. Car culture has conflicted with climate crisis priorities because car culture has typically been an industry that incurs enormous environmental costs. The environmentally negative reputation of companies is ameliorated when ECSR is built into a company's overall business communication strategy. This is crucial for controversial or ostensibly environmentally destructive companies like automobile companies. Take for example the introduction of hybrid vehicles which major car companies began to introduce due to consumer responses, government environmental legislation, and a desire to reduce fuel consumption. The popularity of hybrids like the Toyota Prius is a direct result of the climate crisis forcing controversial companies to respond to their outsized environmental impact. This is why ecologically destructive industries 
like automobile companies are prone to pay more attention to stakeholders perspectives and adopt an information strategy for their ECSR (Vollero, Conte, Siano, and Covucci, 2019, p. 148).

When analyzing automobile companies' use of ECSR it is crucial to understand their history of using nature imagery and motifs in their advertising. Separate from ECSR activities is a long history of green advertising and the use of nature to promote automobiles. Raymond Williams regards the word "nature" as a complex word which has all too often been appropriated by mass media (Hansen, 2002, p. 509). One main critique of using "nature" for ECSR to enhance corporate reputations is that it often contributes to "greenwashing." Greenwashing is "the act of misleading consumers regarding the environmental practices of a company or the environmental benefits of a product or service," (Budinsky and Bryant, 2013, p.209). Deardorff (2010) reports that "one environmental marketing firm surveyed 2,200+ products making positive environmental claims and found some form of greenwashing in $98 \%$ of these communications" (Reilly and Hynan, 2014, p. 2). A variety of studies have shown that public understanding of the word "nature" has been culturally constructed; as a result, natural imagery and beautiful scenes can be used in advertisements to reinforce escapism and can seem like a response to the climate crisis in cultures that view images of nature as promoting eco-consciousness. In Hansen's study, researchers discovered that nature in car advertisements is portrayed most frequently at a distance to isolate the promoted vehicle and to depict it as the only mechanical artifact in its environment (Hansen, 2002, p. 509). Hansen and Machin $(2008,2014)$ explain that there has been an appropriation of the environment by, for example, the automotive industry, such that it serves as a generator of emotional responses that "greenwash" the selling of environmentally destructive products (Chen, 2016, p. 16). 
The appropriation of nature within car advertising has extended globally from the West. For example, China's use of nature in automobile advertisements reinforces the separation between humans and nature by focusing on the car itself and business promotion over real environmental solutions (Chen 2016). Nature is then glorified and made to seem untouched, which is a common theme within green advertising. Budinsky and Bryant (2013) note that the neoliberal agenda disguised by green advertisements promotes individualism and that this contradicts the need for a more sustainable future (Chen, 2016, p. 14). These individual and market-based responses to the climate crisis further separates humans from nature while offering the promise of green capitalism as a solution. Given Tesla's growing popularity within the automotive industry, studying their ECSR is crucial coming from a brand that is fully green and not using any fossil fuels.

Post-environmentalism, a capitalist approach to solving ecological problems (Laser and Stowell 2019), focuses on the responsibility humans and their companies have towards revitalizing and maintaining the natural environment during the climate crisis. This form of green consumerism has emerged as an attempt to enact a circular economy in which companies can address the environmental issues by modifying the processes that go into their products' production. One recent example is Apple's recycling robots created to break down and recycle iPhones. Apple uses a technocratic scheme to deal with waste in support of their ECSR objectives (Laser and Stowell, 2019. p. 1). Additionally, their overall company image is increasingly focused on sustainable solutions to solve environmental challenges. One company in particular, similar to Apple, has gone above and beyond by building sustainability and corporate responsibility into their products from the beginning: Tesla. Tesla is an electric vehicle and clean energy company that has leveraged Twitter using ECSR communication. 


\section{ECSR and Tesla's Twitter}

Companies like Tesla have turned to online tools like Twitter to market their corporate social responsibility and share their story. This is because as the eco-friendly expectations of both stakeholders and consumers grow, the relevance of communicating corporate social responsibility within companies' external and internal communications also grows. As a result of digitization, Social Networking Spaces (SNS) have increasingly been used as a primary tool for companies to reach stakeholders and consumers concerning their environmental mission.

One of the risks of utilizing CSR is that a company may be accused of "greenwashing" and ultimately lead to less trust by consumers and stakeholders alike (Araujo \& Kollat, 2018, p.420). However, the overall impact of corporate storytelling as a form of CSR can also lead to more trust from consumers and stakeholders. In a recent study, scholars conducted studies of eight global brands' use of CSR on Twitter and discovered that there is a lack of co-production of value when tweets are one way or broadcast style (Okazaki, Plangger, West, Menendez 2019). Opposite to this study's findings, I have been noticing a trend in the culture of sharing amongst Tesla's followers which has provided a co-production of value and marketing for Tesla's brand. This suggests to me that Tesla is maximizing their use of Twitter to market their ECSR as well as their overall brand image. 


\section{Research Questions}

This MRP about Tesla's use of ECSR is driven by the following research questions:

1. What is the role of Twitter for Tesla's CSR efforts and what kind of rhetoric are they using for their audience? Is there any correlation between Tesla's CSR tweets and Tesla's share price?

2. Why does Tesla utilize Twitter to market their CSR? What types of messages are they communicating, and why? What tends to be the response to these posts?

3. What does Tesla's social media network look like? Who is connected to this network? How is this working for Tesla and how could they do it better? 
Methodology

This study will use two different methodologies, leading to a mixed methods approach. First, this study will conduct a rhetorical analysis of 104 tweets from Tesla from the random dates March 172019 to March 162020 which will be the main sample used within this study to identify the ways in which Tesla has been persuasive in sharing their environmental mission to their online audience. I will focus on whether or not they utilize images to shape their posts and what the topic of the post are. Understanding that the Tesla ECSR agenda is the main goal, a general rhetorical analysis of both ECSR and non ECSR tweets is needed to identify whether the content is being produced to persuade, inform or entertain the audience. I will also look at the corresponding stock prices compared to each tweet within the same year to analyze whether or not there is a relationship between Tesla's tweets and its stock price. Next, I will devise a content analysis of the same tweets to better understand the ways that Tesla is delivering their information as well as how it is being received. Then, using the top twenty comments of both the ECSR and non ECSR tweets, I will be looking at the way the information is being received by people to understand the levels of engagement (in the form of audience responses to Tesla's tweets). 


\section{Data Collection Steps}

Using digital social research for data capture is crucial in order to understand the culture of online spaces. With the rise of digitization there has also been a rise of new research methods. According to Marres, digital types of methodological innovations include data and traces, interaction and interactivity like conversation analysis, and research design including network analysis (Marres, 2017). This scaling up of network analysis causes a shift in research from modelling networks to analyzing real world dynamics within them. The ability to dissect the interactivity of participants online allows researchers to develop new modes of social inquiry that challenge older methods of research. This rise of digital social research has led to the adaptation of methodologies carefully designed with both device aware and device driven research. This analysis of 'happening content' in digital methods is arguably similar to Latour's Actor Network Theory due to the act of analyzing the dynamics between the actors in the network (Marres \& Gerlitz, 2016, p.25). This interest in 'happening content' allows the digital researcher to have a real time understanding of the cultures formed within online communities. The overall benefit of data capture allows the social researcher to gain an in-depth understanding of the mechanics and relationships formed within online networks and navigate through digital spaces like social media platforms. The data captured within this study will be crucial for understanding how to market environmental corporate social responsibility (ECSR) online.

For the data capturing process, I began with an overview of Tesla's posts, then I decided to focus on the ECSR messaging which is meant to deliver eco-friendly product information. Then, doing an overview of the Tesla Twitter feed and examining the sustainability terms being used, I settled on twelve keywords to analyze in order to categorize a range of ECSR tweet categories. These categories included: environmental customer welfare, environmental community 
involvement, and philanthropy (Rashid, 2014, p.502). The keywords I chose to use based on these categories were: solar, tesla energy, renewable energy, sustainable, green energy, clean energy, climate change, global warming, environmental, environment, sustainable future, and nature. Next, using the Twitter API, I took a total of 28 tweets matched with the 12 keywords over a 1 year span (March 17 2019- March 16 2020) (Appendix A). I compared each matched term with the other tweets posted that day for a total of 104 tweets analyzed with 79 posts being non-ECSR related. Next, I categorized the data to analyze the top 20 comments of each tweet to see if they used images and whether they focused more on sustainability or the product. To prepare for my quantitative and qualitative analysis, the tweet description, post theme, and how the information was received by the audience was added to my final data set. 
Drawbacks

It is important to recognize the drawbacks of this study that may have influenced the results. First of all, examining a year's worth of Tesla's tweets without looking at Elon Musk's Twitter account, since he's a super influencer on Twitter, may have resulted in this study overlooking significant and relevant social media content. There may have been strong connections between Tesla's and Musk's accounts that could have been examined to see the ways in which Tesla utilizes their CEO to leverage their ECSR and that could have contributed to a deeper understanding of the operations of the company. As for the connection between ECSR tweets and the stock market, some people may have an impression that they do influence stock prices, but evidence from this study suggests that there is no connection. Including this data without a clear understanding of the stock market or information on Elon Musk's influence impacts this study. Finally, the environmental terms chosen in this example only represent a small sample, thus the 12 keyword terms utilized to form the analysis of Tesla's ECSR tweets do not represent Tesla's full range of ECSR communication. 
Rhetorical analysis

In order to understand the role of Twitter for Tesla's environmental ECSR efforts, it is vital to recognize how the platform is being used by dissecting the motivation behind the tweets and what they represent. In order to do so, one can conduct a rhetorical analysis, or the unpacking of discourse, to illuminate its persuasive power (Leach, 2000, p. 207). Politicians and lawmakers are known to be well crafted rhetorical speakers who use words strategically to convey information in their favour. Similarly, companies and CEOs utilize these rhetorical tactics to benefit sales and create a sense of community amongst their followers and customers. With rhetorical analysis, the “'intentions' of authors can be divined by their texts or oral performances" (Leach, 2000, p. 210). Utilizing Twitter, this study's rhetorical analysis seeks to uncover the intentions of Tesla through their tweets.

The following rhetorical analysis analyzes the captured data to understand the ways in which Tesla's discourse is being shaped for their audience on Twitter. The goal of this rhetorical analysis is to compare ECSR tagged terms to non-tagged terms to understand the ways in which ECSR is being communicated through Tesla compared to their ordinary daily tweets. Do both convey information, persuade, or entertain their audience and what is the difference? The importance of comparing the two types of tweets is a critical aspect of this research and will be the main agenda of the following rhetorical analysis.

Overall, analyzing the rhetoric of the tweets based on the post theme within the sample data set has shown that the main discussions surround both Tesla's mission and its charging technology known as "supercharging." Specifically, ECSR tagged tweets discussed solar charging as well as Tesla's mission and supercharging. What this shows is that Tesla is intentional in their communication surrounding ECSR because they focus on their 
environmentally friendly alternatives (i.e. electricity) to gasoline as fuel. One example of a tweet under the ECSR tagged keyword solar was, "join us at 2pm PT today to learn all about our V3 Solar Roof product. Visit https://t.co/rd0ILdpzRL to listen in” (October 25, 2019) (Appendix B). Here, the company is inviting the audience to gain more information about their product. The rhetoric is to inform followers about the product rather than to persuade or entertain. Petrov notes that a product "represents itself both intrinsically and as a symbolic construction that is based on culture and an orientation to cultural values" (2016, p. 30). In other words, Tesla's solar product acts as a symbol for conscious consumerism.

When looking at the post theme of non-tagged tweets, the data uncovered that they primarily discussed humour, Teslas in boring places, and the customer referral program as well as supercharging and Tesla's mission. This data uncovers that Tesla is specifically utilizing rhetoric in their daily tweets to entertain and inform. One example of a tweet on May 10, 2019 stated, "if dogs could talk - and we cannot stress this enough - they would tell you to drive a Tesla" (Appendix C). This use of humorous imagery is a common theme for Tesla. In addition to humour, “the@Tesla account uses a more professional style of discourse, emphasizing the purification of the company's image" (Schumacher, 2019, p. 27). Like their ECSR tagged tweets, the company is utilizing Twitter to promote their image as an environmentally conscious company.

The sample data set also looked at whether or not tweets would use images to convey value to their consumers. This study has discovered that Tesla is infamous for utilizing images, threads, and videos in their tweets. One study looked at Tesla's tweet of their Model S on a beautiful hill which stated "thank you for driving towards a more sustainable future". This tweet they discovered, "sells a Model S to the consumer; on a deeper level, Tesla sells the idea of a brighter 
tomorrow through an environmental utopia without carbon emissions" (Petrov, 2016, p. 30). Here, Tesla is outwardly utilizing images of nature in connection with their energy efficiency to sell to the conscious consumer. This can be further explored within the data sample where the study discovered that most tweets posted have an image. Specifically, analyzing the tweets with the ECSR terms, the data found that more of Tesla's tweets utilize images than not (see Appendix D).

Furthermore, Tesla's non-tagged tweets are more likely to have images (see Appendix E); however there are more tweets without images than there are of the ECSR tagged tweets. In other words, ECSR tagged tweets are more likely to utilize images than plain text. Strikingly, there is a connection between the use of images and the volume of favourites and retweets in ECSR tagged tweets. On average, as discussed in the next section (Content Analysis), there are more favourites and retweets on ECSR tagged tweets and these tweets are more likely to use images. This shows that use of images when conveying environmental information will be successful for companies communicating their ECSR on Twitter.

Overall, "Twitter is a medium of immediacy, information, and interactivity" (Johnson, 2012, p.57), and "because of Twitter's community building nature, Twitter is situational and rhetorical" (Johnson, 2012, p.62). Therefore, Twitter has provided Tesla with an excellent tool to utilize to construct their company's overall brand image.

\section{Relation to Stock Price}

When a company has constructed a compelling brand image, the stock market may be influenced by the company's social media use. One study discovered that rhetoric surrounding emotions directly affected the price of the stock market that day (Zhang, Fuehres, and Gloor, 2011, p. 60). This study conducted an analysis of the stock prices in relation to the rhetoric used 
that day. Utilizing the above data set, I examined the stock prices for each day studied and by looking at the highest and lowest closing prices discovered a lot about the company's communication strategy.

The average stock price from the pulled ECSR keywords in this sample was \$291.41. In these tweets, the average post theme was on the solar roof product and Tesla's mission where the rhetoric is to inform the audience. The highest closing stock price of $\$ 858.40$ was on Sunday February 16, 2020 when Tesla tweeted, "Solar Roof is taking off across the US. Become a Solar Roof installer today, no experience needed!" with a video of Tesla's solar roofing job. This was the only tweet that day gaining 28176 favourites and 3847 retweets. Tesla's post about how their product is successful arguably aided them in raising the stock price that day, though there is not enough evidence to prove that the tweet was solely responsible for the raised price. On the other hand, the lowest closing stock price of $\$ 179.97$ was on June 1st 2019 when Tesla tweeted, “At Tesla, we support a diverse, inclusive and safe environment for all," with an image of people walking beside Model X wrapped in LGBTQ colours stating "Walking into pride month like..." where the remaining tweets the same day were also promoting Tesla's support of LQBTQ pride. There is not enough evidence to prove that Tesla's support of pride month online correlates to a lower stock closing price because these tweets took place on the weekend and could have dropped from an event the previous day. In all of the pulled tweets, the main objective or intention that Tesla's rhetoric uncovered is their dedication to informing their audience which can have a slight impact on the stock price that day. Unfortunately, this data set does not give enough evidence to prove that there is a direct relationship between ECSR tweets and Tesla's stock market prices. However, the pursuit of image building, which is related to stock prices, is a key driver for Tesla's ECSR activities. 


\section{Content Analysis}

In order to understand why Tesla is promoting their environmental corporate social responsibility (ECSR) on Twitter, it is vital to recognize both the messages they are sharing and the types of responses being given. By conducting a content analysis, this study will use the same data sample used in the rhetorical analysis to examine the content being shared by Tesla's Twitter account to understand how Twitter is being leveraged by the company and how ECSR can be communicated on Twitter. By delving into a qualitative analysis of the number of favourites and retweets, the post theme, and how the post was received through an inquiry of the comments section, this research seeks to understand the overall strategy of Tesla's Twitter use and how the responses influence or hinder their credibility as a company.

First, it is vital to understand why Twitter is such an essential tool for Tesla to use for promoting ECSR. Social media itself allows for new levels of engagement because "companies and audiences are able to form a participatory relationship" (Schumacher, 2019, p. 11). After examining Tesla's twitter content over the course of a year, it is clear they have formed a strong participatory relationship with their audience based on the responses to their tweets. What is extremely interesting is how they have been successfully subliminally marketing their ECSR on Twitter even though "Tesla has publicly claimed it does not have a marketing department” (Petrov, 2016, p. 23). This tactic has allowed Tesla to create a strong sense of community amongst its followers without an enormous ad campaign and accompanying budget.

This study examines the responses to Tesla's tweets to understand the conversations being held surrounding ECSR and to see if the message is being organically reciprocated by the community. Utilizing the data sample, this study begins by examining the response towards both ECSR tagged tweets and non-ECSR tagged tweets by examining both retweets and favourites. The 
data set shows that ECSR tagged tweets have 7540 favourites while non-tagged tweets only have 4390 favourites. In addition, ECSR tagged tweets have 1031 retweets while non-tagged tweets have 399 retweets. Here, the study discovered that on average, there are more favourites and retweets on sustainability ECSR tagged tweets than non-tagged tweets (See Appendix F).

This data shows that although there are fewer ECSR tweets within the year, there is more audience interaction and focus on these tweets than an average post. This also shows that Tesla has been successful leveraging Twitter to communicate and share information regarding sustainability on this platform.

When examining the tweets' overall responses within the data, it was discovered that in ECSR tagged tweets, $53.2 \%$ of the conversations being held on Twitter's post were focused on Tesla's products while only 5\% were focused on sustainability (See Appendix G). This accurately reflects my hypothesis where I proposed that more conversations would be about the Tesla product than about sustainability. Compared to the tweets tagged with the ECSR terms, the non-tagged tweets sampled from the same dates show that $56.4 \%$ of the conversations being held were about other topics, while $41.7 \%$ were about the product, leaving $1.9 \%$ for conversations about sustainability (See Appendix H). This data is useful in describing how Tesla's tweets even when random create productive discussions about the products and therefore create free forms of advertisement for the company. This data also shows that on average there are more discussions surrounding sustainability within the ECSR tagged tweets.

Next, analyzing how the information was received by ECSR tagged tweets, the data uncovered that instead of sharing information about climate change the conversations were primarily about sharing facts about Tesla in regards to sustainability, and discussing product likes and dislikes. One important discovery was that the majority of Tesla's ECSR tagged tweets had 
comments that were a thread of tweets from Tesla. This technical affordance offered by Twitter is valuable in conveying extra information within the 140 character limit Twitter has set and allows Tesla to take up the primary space in the comments section to attract attention. The thread allows Tesla to "link to a Web site or a blog where the (company) can share video, audio, text, and photographs with the audience, which creates a more intimate connection" (Johnson, 2012, p.56). This allows Tesla to claim a majority of the comments section to force the community to navigate through their information before reading other responses by other accounts. Tesla is known for their pinned thread tweets that share information about Tesla's mission. Tesla allows people to come together to discuss their products which allows the average consumer to not only be in close communication with Tesla, but be a part of a larger community, specifically through the sharing of personal experiences with the product. The data sample uncovered a huge trend in the sharing of experiences and photos with the product in the comments section. Studies have shown that "retweeting positive customer experiences is a unique feature of Twitter" (Schumacher, 2019, p. 29) which has allowed Tesla to have access to free marketing by creating a platform for their consumers to share information.

On the other hand, when analyzing how the information was received, the data discovered that non-ECSR tagged tweets sparked a meme culture and emoji sharing culture, discussion of Tesla's mission, discussion of Tesla's new sarcastic Twitter usage, and people sharing their customer referrals online. One date, May 09, showed most posts being about sharing pictures of Tesla vehicles in boring places emphasizing the culture of sharing Tesla is creating on Twitter(Appendix I). In addition to the culture of sharing, there is also a culture of humour amongst Tesla's followers. The space Twitter has provided Tesla to use has been turned into a stomping ground for memes. Memes are multimodal and intertextual online content containing multiple 
forms of visual text including an image and caption or movement in a GIF and encourage a participatory response that encourages virality. Often humorous in nature, the meme becomes a vehicle for communicating ideas online concerning specific localized topics (Borzsei, 2013, p. 6). This "glocalization" that the internet allows enables the Tesla community to share humorous photos and concepts on the digital space offered by Twitter (Borzsei, 2013, p. 20). Using the data sample chosen, Tesla's Twitter page offers a great example of how the comments section of a post can turn into a community revolving around meme culture. On May 10 2019, Tesla posted tweets about dogs driving Tesla vehicles, and as a response was flooded with comments of other people sharing images of their dogs, some even in Tesla's frunk feature (see Appendix J). This sharing of humorous content creates a sense of community amongst Tesla's followers while subliminally offering free marketing for the Tesla brand through the participatory sharing of photos of Tesla's vehicles. Another interesting data set in the sample showed that two consumers put in a customer complaint to ask Tesla a question that went unnoticed, while on the other hand someone shared a photo of a Tesla meme and Tesla reposted it immediately. This shows that Tesla has created a space of sharing product promotions and humorous posts versus customer concerns on this platform.

Through this content analysis, it is interesting that the ECSR tweets are used to inform the audience, while the primary focus of non-tagged tweets is to entertain, then inform. This shows that Tesla's non-tagged tweets have some power in influencing the ECSR posts as they provide a sort of "break" to the critically informative content to enable acts of participatory culture. In doing so, when a relatively serious post is made, the tweet will gain a more intense response because it is different than the tweets that primarily entertain the users. Here, Tesla demonstrates a successful example of how a company may leverage a social media platform to enable a higher response for 
the content they desire the audience to receive most which is arguably information about their environmental mission as a company. They do this by creating a space on Twitter for a community to form through sparking both informational and humorous conversation in their tweets. 


\section{Conclusion}

What is especially interesting about Tesla is that their vehicles, in addition to being a consumer product, have also become icons for consumer activists. It is also intriguing how Tesla chooses to shape the brand through their sarcastic rhetoric. All of this, however, is not surprising since texts "are multi-functional" and "influence ways of acting, ways of representing and ways of being" (Brei and Bohm, 2014, p.11). The texts that Tesla utilizes for the brand's image are often humorous, sarcastic, or informative, all of which have influenced the culture of their brand. Their following is tight knit because Tesla itself acts as a consumer activist brand and Twitter offers a place to input interdiscursive content concerning the product.

Tesla's Twitter, as shown through the content analysis of the comments, is an online community for its users. This is reminiscent of Anderson's "imagined communities" where "the members of even the smallest nation will never know most of their fellow-members" (Gruzd, Takhteyev, and Wellman, 2011, p. 1297), yet a common language and sense of temporality brings people together. Tesla's online following could also be described as a psychological brand community (PSBC). PSBCs are bolstered by "the degree to which individuals identify with (a) the brand itself and (b) the group of all individuals who purchase or utilize that brand" (Carlson, Suter, and Brown, 2008, p.286). As the data shows, Twitter offers Tesla an imagined community for its users to connect with one another as well as the company. Additionally, this online community offers a free form of advertisement which helps the Tesla brand create long term relationships with its consumers.

The community of Tesla followers on Twitter have created a sort of system in which the overall experience is co-created. The data uncovered that the engagement of Tesla's followers in the comments section on Twitter focus primarily on informing other members about the Tesla 
products, experiences with the products, or simple humorous and light conversation. These conversations are rooted by the Tesla brand and sprout to form strong levels of engagement and trust amongst their followers.

Since this project relied on both rhetorical and content analysis to research the data sample, there are many connections between the results. What is interesting is how the rhetoric being utilized shapes the content being formed to create a relationship between the company and consumer concerning ECSR. This study discovered that there were more favourites and retweets on ECSR tagged posts where discussions were mainly surrounding Tesla's mission and supercharging. On the other hand, non-tagged tweets were primarily offering humorous or sarcastic discourse to the audience. The key difference that the rhetorical and content analysis in combination uncover is that ECSR posts are used to inform and discuss the product, while nontagged posts are used primarily to entertain.

This study discovered that on average, CSR tagged tweets gain more favourites and retweets than an average post. This shows that posts regarding sustainability are being received better than average posts by Tesla. This data also suggests that Tesla has been successful marketing their CSR initiatives through Twitter to gain stronger relationships with followers. Furthermore, with both ECSR tagged tweets and non-tagged tweets, there are a large amount of discussions being made about the Tesla products. This shows that Tesla has leveraged a space of collaborative consumption which has created free advertising and promotion for Tesla.

From a qualitative perspective, this data shows a trend in the culture of sharing amongst Tesla's followers which has provided a co-production of value and marketing for Tesla's brand. This is because social media companies like Twitter act like platforms with which to coordinate collaborative consumption and communicative sharing of information (John, 2017, p.71). Here on 
Twitter, followers would share their personal experiences with Tesla, photos of their own Tesla or discussing the Tesla products which offer a form of free online advertisement for the company. Specifically I have noticed a lot of posts to be humorous and encouraging people to share photos and memes. The sense of belonging that sharing brings is part of a communicative turn that Twitter as a platform has leveraged. Sharing is social and contagious and companies recognize the value of creating spaces to share. This becomes a networked affect where people engage in both sending and receiving while creating meaning and producing values through images and text. Arguably Tesla has leveraged Twitter to market their ECSR as well as overall brand image simply by providing a space to share ideas and memes. The findings within this study show that this sharing behaviour has been leveraged by Tesla to create a sense of belonging and meaning making for Tesla's overall mission.

The aim of this study is to educate communication professionals about the benefits of using Twitter to market ECSR online. This information can benefit corporations both financially and psychologically as "customers could be a valuable source of information for companies" (Laroche, Habibi, Richard, and Sankaranarayanan). As the data uncovers, the use of Twitter allows companies to gain valuable information about their customers' overall experiences with the products through the comments section. Not only does Tesla's interaction with followers on Twitter increase brand awareness, it also ignites and reinforces brand loyalty and Tesla's efforts to market itself as a company that prioritizes ECSR. 


\section{References}

Araujo, T., \& Kollat, J. (2018). Communicating Effectively about CSR on Twitter: The Power of Engaging Strategies and Storytelling Elements. Internet Research, 28(2), 419-431. doi:10.1108/IntR-04-2017-0172

Börzsei, L. K. (2013). Makes a Meme Instead. The Selected Works of Linda Börzsei, 1-28.

Brei, V., \& Böhm, S. (2014). '1L=10L for Africa': Corporate Social Responsibility and the Transformation of Bottled Water into a 'Consumer Activist' Commodity. Discourse \& Society, 25(1), 3-31. https://doi.org/10.1177/0957926513503536

Bubna-Litic, K. (2007). Climate Change and Corporate Social Responsibility: The Intersection of Corporate Environmental Law. Environmental and Planning Law Journal.

Budinsky, J., \& Bryant, S. (2013). "It's Not Easy Being Green": The Greenwashing of Environmental Discourses in Advertising. Canadian Journal of Communication, $38(2)$.

Carlson, B. D., Suter, T. A., \& Brown, T. J. (2008). Social Versus Psychological Brand Community: The Role of Psychological Sense of Brand Community. Journal of Business Research, 61(4), 284-291.

Chen, S. (2016). Selling the Environment: Green Marketing Discourse in China's Automobile Advertising. Discourse, Context \& Media, 12, 11-19. https://doi.org/10.1016/j.dcm.2016.03.003 
Gharios R., Yahchouchi Abi Chaker L. (2020) Social Media and Corporate Social Responsibility. In: Azoury N., Daou L. (eds) Business and Social Media in the Middle East. Palgrave Macmillan, Cham. https://doi.org/10.1007/978-3-03045960-4_2

Gruzd, A., Wellman, B., \& Takhteyev, Y. (2011). Imagining Twitter as an Imagined Community. American Behavioral Scientist, 55(10), 1294-1318.

Hansen, A. (2002). Discourses of Nature in Advertising. Communications, 27(4), 499511. https://doi.org/10.1515/comm.2002.005

Hollebeek, L. D., Juric, B., \& Tang, W. (2017). Virtual Brand Community Engagement Practices: A Refined Typology and Model. Journal of Services Marketing.

John, N. A. (2017). The Age of Sharing. Polity Press.

Johnson, J. (2012). Twitter Bites and Romney: Examining the Rhetorical Situation of the 2012 Presidential Election in 140 Characters. Journal of Contemporary Rhetoric, 2. Laroche, M., Habibi, M. R., Richard, M. O., \& Sankaranarayanan, R. (2012). The Effects of Social Media Based Brand Communities on Brand Community Markers, Value Creation Practices, Brand Trust and Brand Loyalty. Computers in Human Behavior, 28(5), 1755-1767.

Laser, F. \& Stowell A. (2019). Thinking like Apple's Recycling Robots: Toward the Activation of Responsibility in a Post Environmentalist World. Ephemera. http://www.ephemerajournal.org/contribution/thinking-apple\%E2\%80\%99srecycling-robots-toward-activation-responsibility-postenvironmentalist 
Leach, J. (2000). Rhetorical Analysis. Qualitative Researching with Text, Image and Sound, 207-226.

Marres, N. (2017). Do We Need New Methods? Digital Sociology, London: Polity.

Marres, N., \& Gerlitz, C. (2016). Interface Methods Renegotiating Relations between Digital Social Research, STS and the Sociology. The Sociological Review, 64(1), 21-46. doi:10.1111/1467-954X.12314

Okazaki, S., Plangger, K., West, D., \& Menéndez, H. D. (2019). Exploring Digital Corporate Social Responsibility Communications on Twitter. Journal of Business Research, S0148296319305259. https://doi.org/10.1016/j.jbusres.2019.09.006

Petrov, S. (2016). From Insane to Ludicrous: Electric Cars, Environmental Communication, and Cultural Distinction.

Rashid, N. R. N. A., Rahman, N. I. A., \& Khalid, S. A. (2014). Environmental Corporate Social Responsibility (ECSR) as a Strategic Marketing Initiatives. Procedia Social and Behavioral Sciences, 130, 499-508. https://doi.org/10.1016/j.sbspro.2014.04.058

Reilly, A. H., \& Hynan, K. A. (2014). Corporate Communication, Sustainability, and Social Media: It's Not Easy (Really) Being Green. Business Horizons, 57(6), 747-758. https://doi.org/10.1016/j.bushor.2014.07.008

Reilly, A. H., \& Larya, N. (2018). External Communication About Sustainability: Corporate Social Responsibility Reports and Social Media Activity. Environmental Communication, 12(5), 621-637. https://doi.org/10.1080/17524032.2018.1424009 
Schumacher, L. (2019). How Do Organizations in the Automotive Industry Make Use of Discursive Strategies in the Arena of Social Media to Retain Legitimacy in Times of Crisis?

Vigneau, L., Humphreys, M., \& Moon, J. (2015). How Do Firms Comply with International Sustainability Standards? Processes and Consequences of Adopting the Global Reporting Initiative. Journal of Business Ethics, 131(2), 469-486.

Vollero, A., Conte, F., Siano, A., \& Covucci, C. (2019). Corporate Social Responsibility Information and Involvement Strategies in Controversial Industries. Corporate Social Responsibility and Environmental Management, 26(1), 141-151. https://doi.org/10.1002/csr.1666

World Bank International Finance Committee, Corporate Social Responsibility. (2009). http://www.ifc.org/ifcext/economics.nsf/content/csr-intropage

Zhang, X., Fuehres, H., \& Gloor, P. A. (2011). Predicting Stock Market Indicators Through Twitter "I Hope it is not as Bad as I Fear". Procedia-Social and Behavioral Sciences, 26, 55-62. 
Appendix

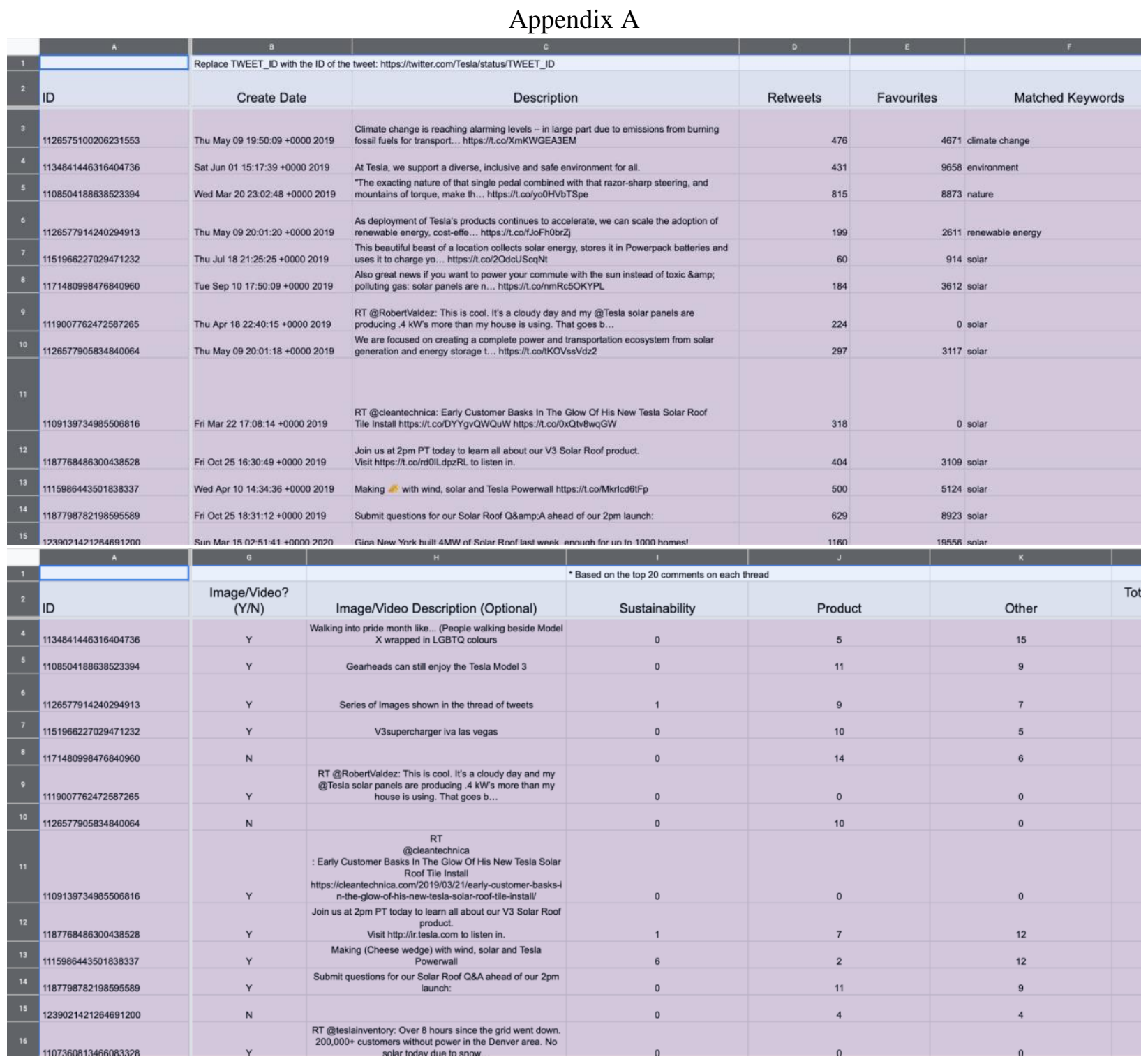

A visual representation of the data sample used within this study. Above: This study focused on

looking at the date of the tweet, the description, retweets and favourites of each tweet as well as the ECSR keyword used. Below: The sample also analyzed if each tweet had an image or video, the description of the tweet, and the focus of the tweet. 
Appendix B

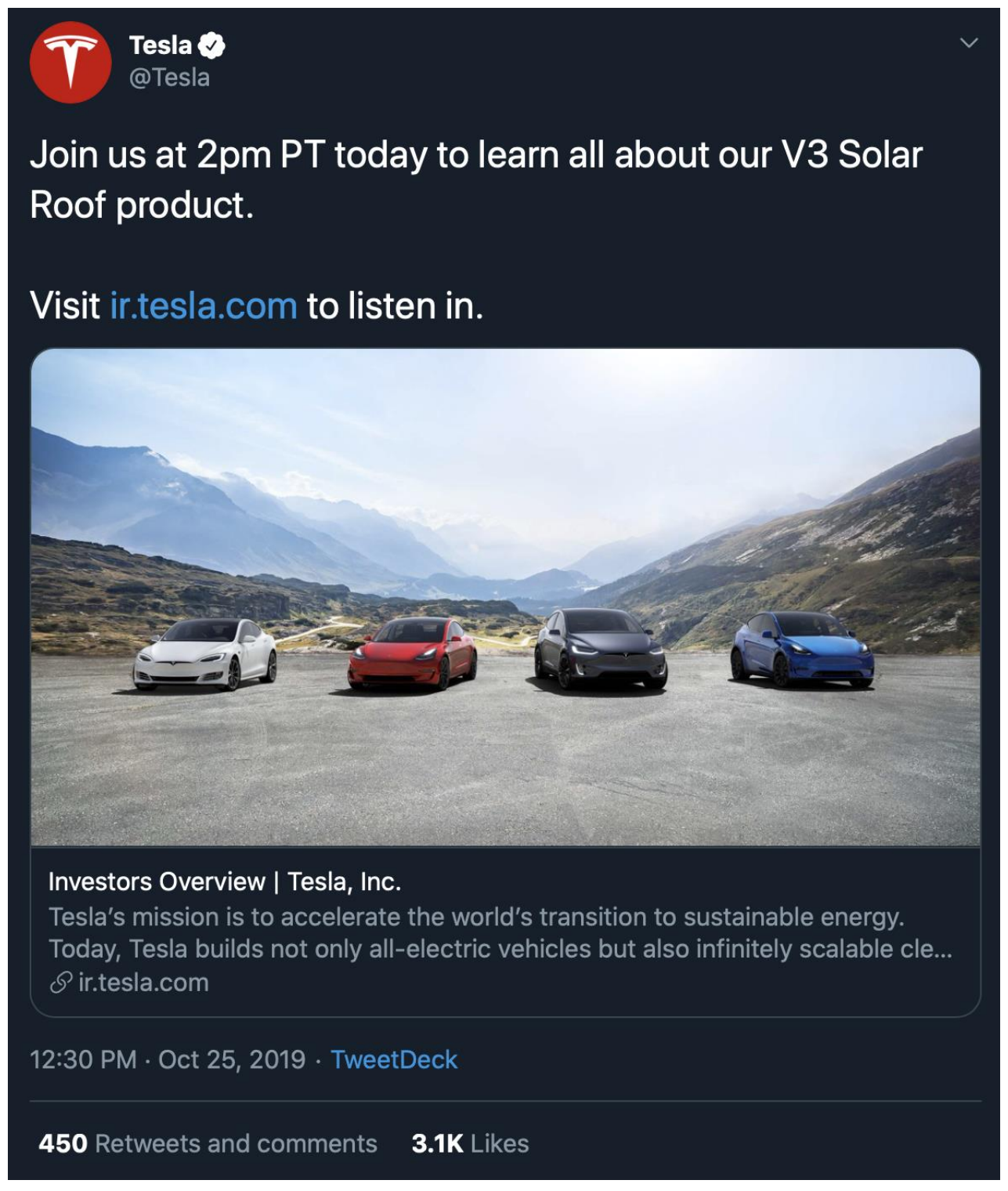

A screenshot of the following tweet promoting their solar roof product. Notice here the imagery of the Tesla cars in nature to portray the connection between Tesla and sustainability. 
Appendix C

Tesla Q

@Tesla

if dogs could talk - and we cannot stress this enough they would tell you to drive a Tesla

2:07 PM · May 10, $2019 \cdot$ TweetDeck

5K Retweets and comments $\quad \mathbf{3 7 . 1 K}$ Likes

A screenshot of the following tweet by Tesla.

Appendix D

Image No Image

20

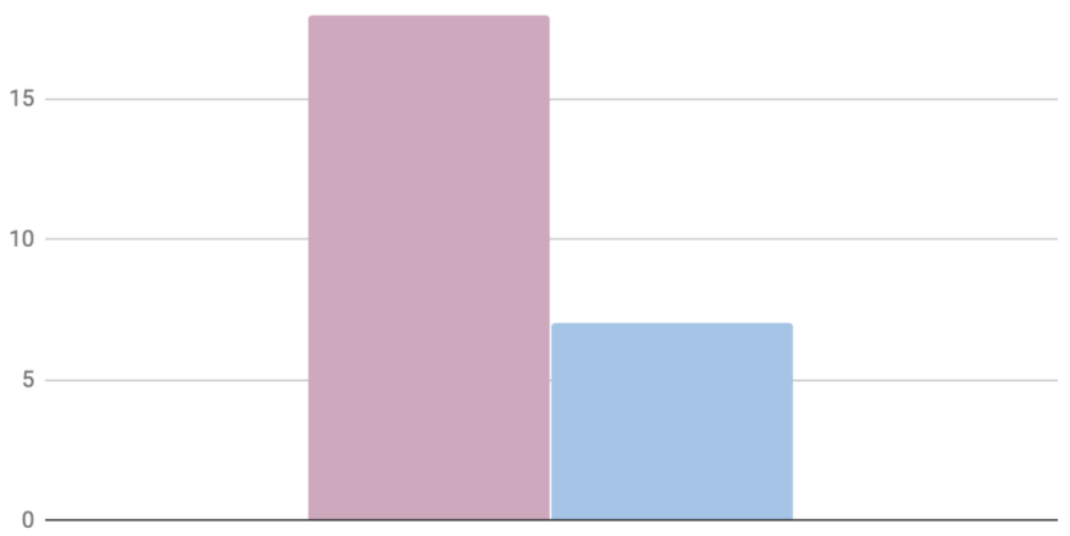

A bar chart depicting ECSR tweets that use images (pink) versus no images (blue). 


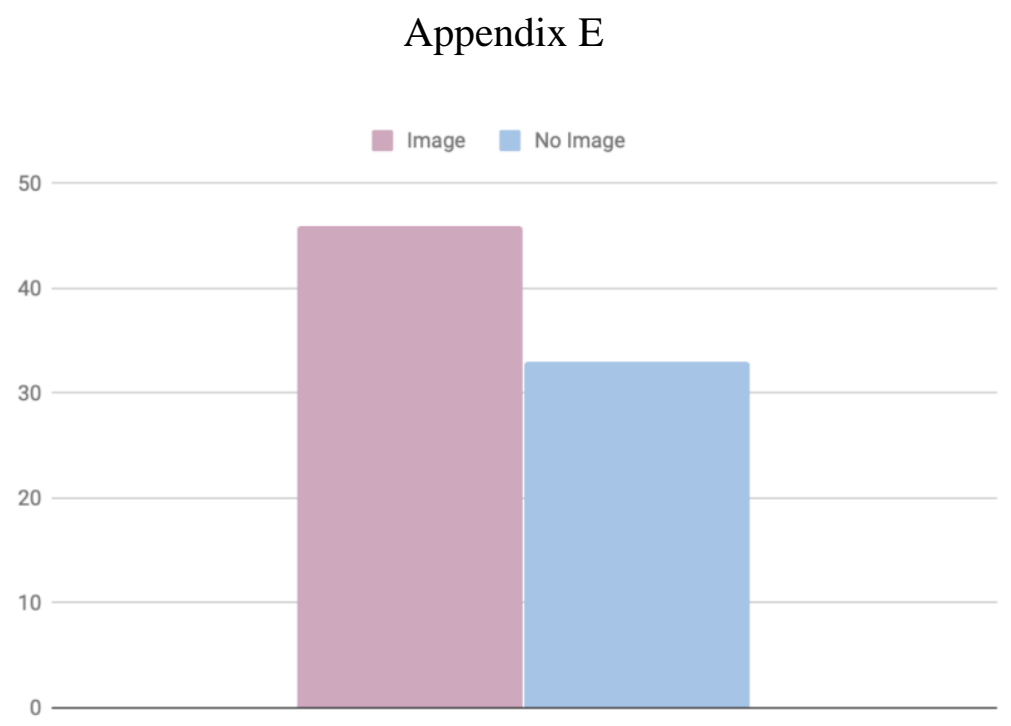

A bar chart depicting non-tagged tweets that use images (pink) versus no images (blue).

\section{Appendix F}

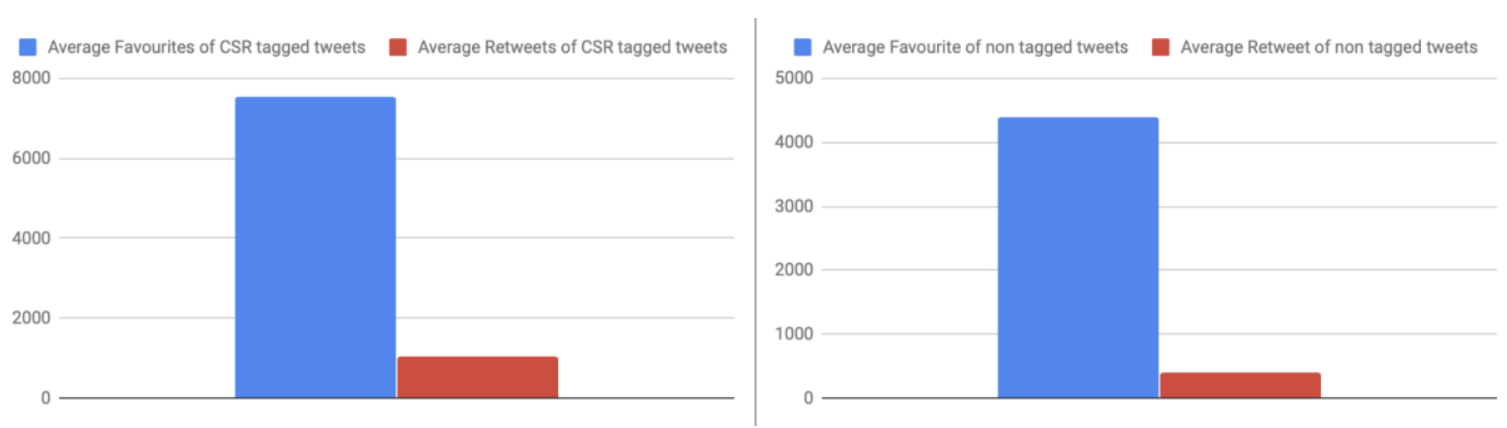

Left: Average of ECSR tagged tweets Favourites in blue, Average of ECSR tagged tweets retweets in red.

Right: Average of non-tagged tweets Favourites in blue, Average of non-tagged tweets retweets in red. 


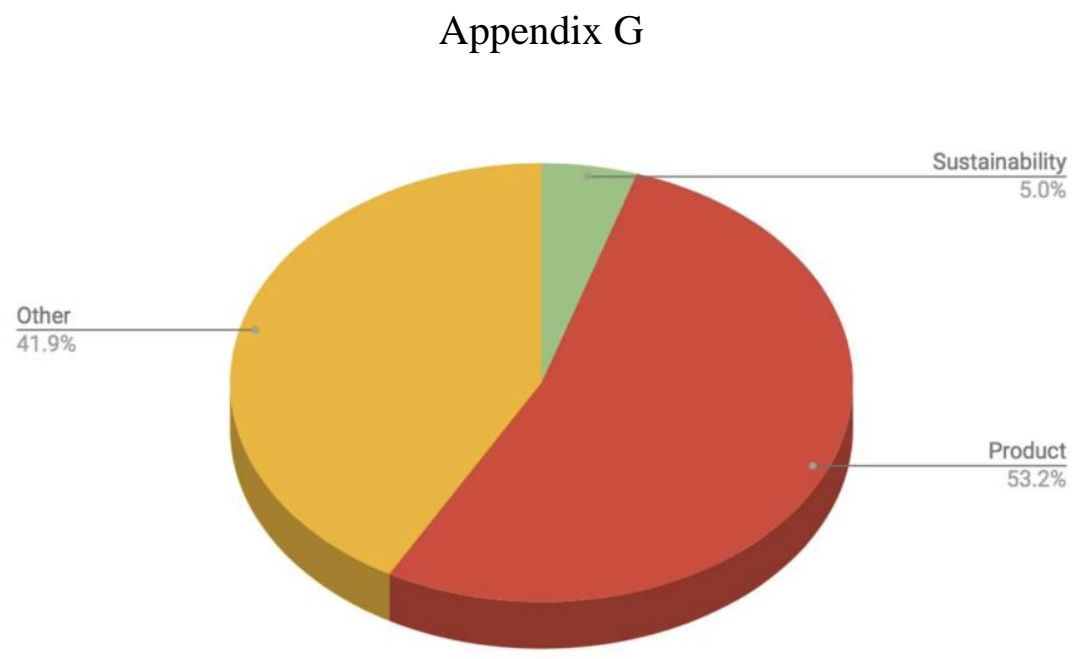

A pie chart showing the categories of the conversations being held for ECSR tagged tweets.

Appendix $\mathrm{H}$

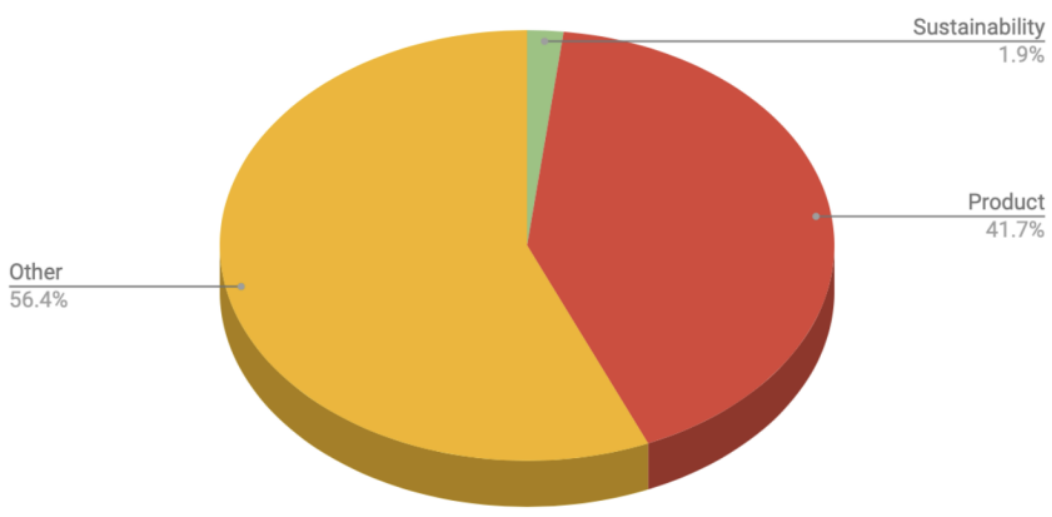

A pie chart showing the categories of the conversations being held for non- tagged tweets. 


\section{Appendix I}

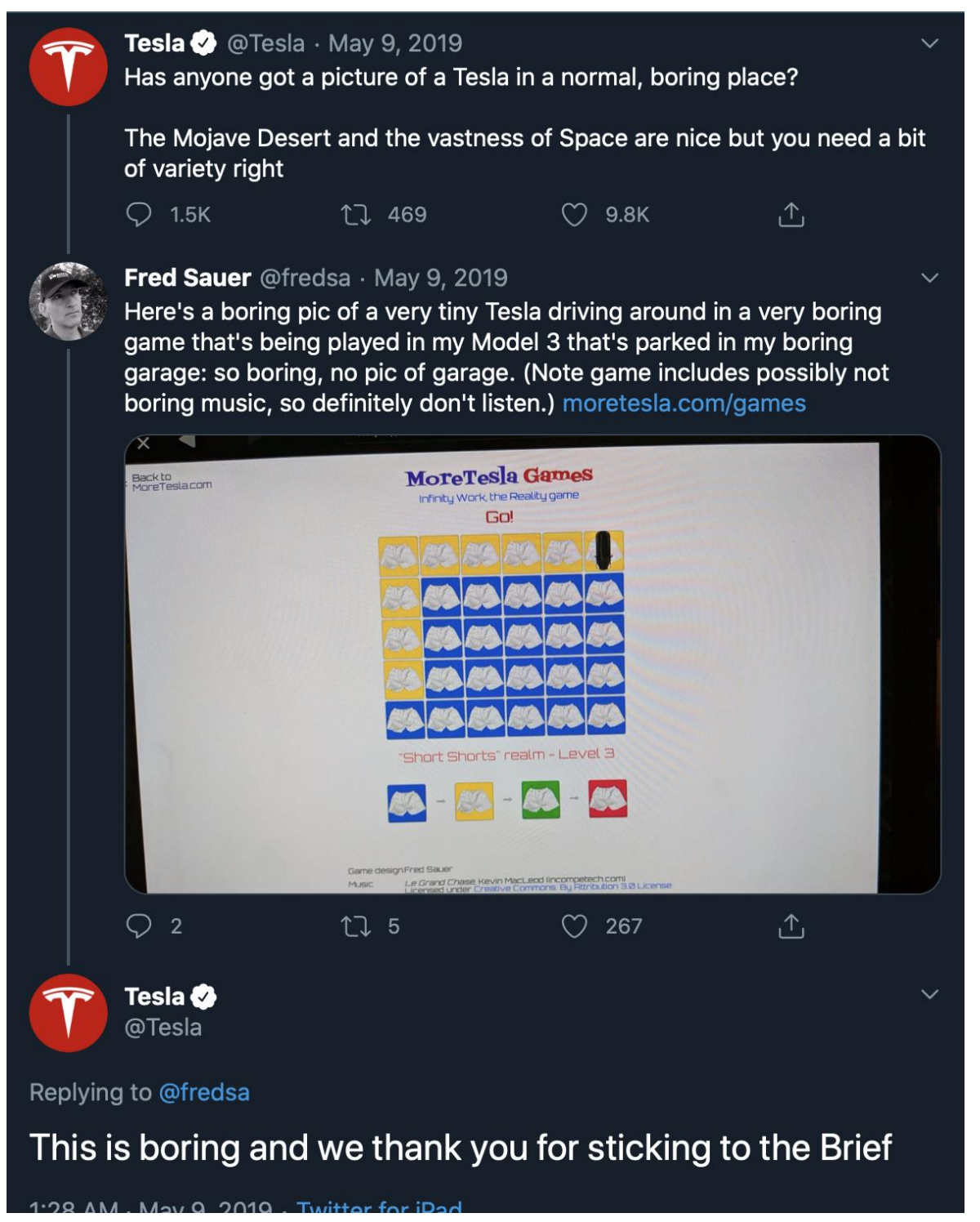

A screenshot of Tesla's tweet asking users to share pictures of Teslas in boring places. 
Appendix $\mathbf{J}$

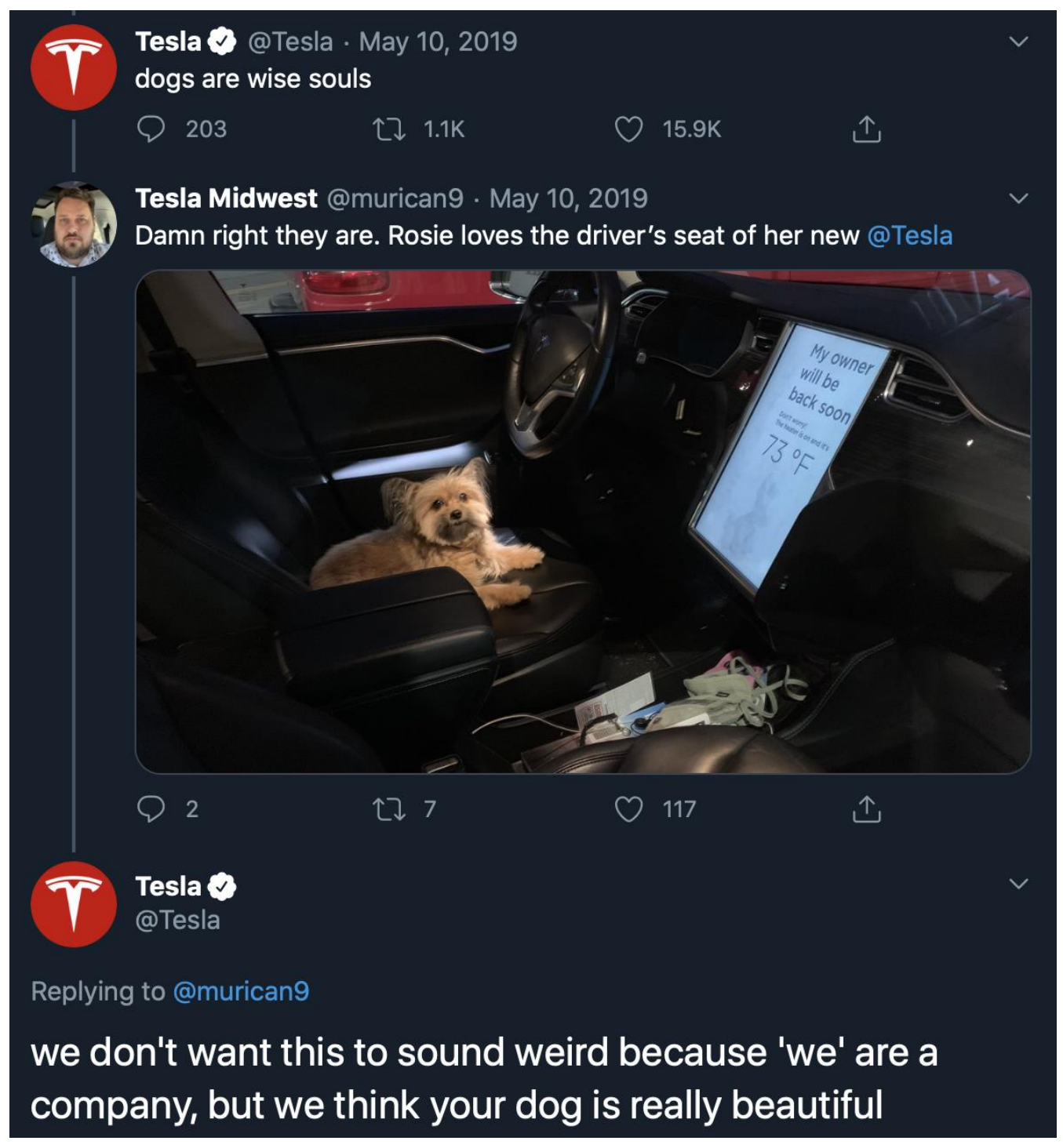

An example of one of Tesla's more humorous tweets. 